\title{
Can continuous scans in orthogonal planes improve diagnostic performance of shear wave elastography for breast lesions?
}

\author{
Pan Yang, Yulan Peng*, Haina Zhao, Honghao Luo, Ya Jin and Yushuang He \\ Department of Ultrasound, West China Hospital, Sichuan University, Chengdu, Sichuan, China
}

\begin{abstract}
.
BACKGROUND: Static shear wave elastography (SWE) is used to detect breast lesions, but slice and plane selections result in discrepancies.

OBJECTIVE: To evaluate the intraobserver reproducibility of continuous SWE, and whether quantitative elasticities in orthogonal planes perform better in the differential diagnosis of breast lesions.

METHOD: One hundred and twenty-two breast lesions scheduled for ultrasound-guided biopsy were recruited. Continuous SWE scans were conducted in orthogonal planes separately. Quantitative elasticities and histopathology results were collected. Reproducibility in the same plane and diagnostic performance in different planes were evaluated.

RESULTS: The maximum and mean elasticities of the hardest portion, and standard deviation of whole lesion, had high inter-class correlation coefficients $(0.87$ to 0.95$)$ and large areas under receiver operation characteristic curve $(0.887$ to 0.899$)$. Without loss of accuracy, sensitivities had increased in orthogonal planes compared with single plane (from $73.17 \%$ up to $82.93 \%$ at most). Mean elasticity of whole lesion and lesion-to-parenchyma ratio were significantly less reproducible and less accurate.

CONCLUSION: Continuous SWE is highly reproducible for the same observer. The maximum and mean elasticities of the hardest portion and standard deviation of whole lesion are most reliable. Furthermore, the sensitivities of the three parameters are improved in orthogonal planes without loss of accuracies.
\end{abstract}

Keywords: Shear wave elastography, quantitative analysis, breast lesion, diagnostic performance

\section{Introduction to SWE}

Shear wave elastography has been proven as an effective method to distinguish malignant from benign lesions [1-4]. The technique depends on the phenomenon that malignant lesions is usually harder than benign lesions [5]6]. SWE works by inducing shear waves that travel faster in harder tissue. Then a very fast acquisition sequence is used to capture the propagation of the shear waves [7]. Accordingly, the difference in stiffness is presented as speed (in m/s) or as Young's modulus (in $\mathrm{kPa}$ ). Recently, several studies have shown that SWE is efficient and highly reproducible for breast masses. In quantitative analysis of SWE, the area under ROC curve have reached 0.962 with maximum elasticity and ICC is up to 0.87 with mean elasticity. These studies are based on three separate static SWE images in longitude

\footnotetext{
${ }^{*}$ Corresponding author: Yulan Peng, Department of Ultrasound, West China Hospital, Sichuan University, Wai nan guo xue xiang 37, Chengdu 610041, Sichuan, China. Tel.: +86 18980601606; E-mail: yulanpeng@163.com.
}

0928-7329/15/\$35.00 (c) 2015 - IOS Press and the authors. All rights reserved

This article is published online with Open Access and distributed under the terms of the Creative Commons Attribution NonCommercial License. 


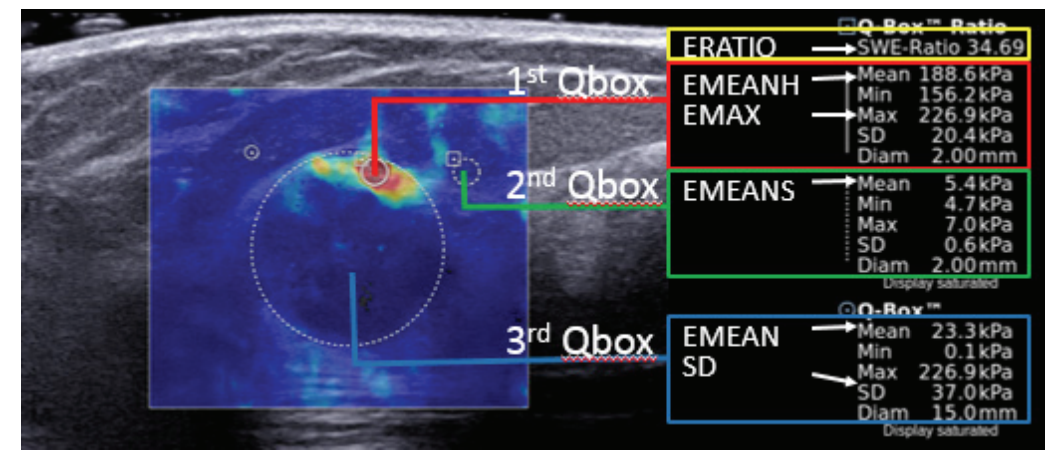

Fig. 1. Placements of Qboxes and parammeters for quantitative elasticity analysis. On the SWE image was an adenoid cystic carcinoma. The quantitative elasticity: EMAX $226.9 \mathrm{kPa}$, EMEANH 188.6 kPa, EMEANS $5.4 \mathrm{kPa}$, ERATIO 34.69, EMAN $23.3 \mathrm{kPa}$, SD $37.0 \mathrm{kPa}$.

planes. However, suspicious features would have been not sufficient in single plane, and the resulting differences in slice selection across acquisitions are likely to affect the study results. So real-time image acquisition and orthogonal planes are recommended [4 [8].

Therefore, the purpose of this study is to evaluate the intraobserver reproducibility of real-time SWE examination, and whether quantitative elasticity in orthogonal planes perform better in the differential diagnosis of breast lesions.

\section{Patients and method}

\subsection{Patients}

The study was performed from July 2014 to January 2015 in West China Hospital, China. The inclusion criteria were female patients with breast mass in B-mode ultrasound and scheduled for ultrasoundguided biopsy. Some patients were exclude if they were: pregnant or breast-feeding, with mass greater than $3 \mathrm{~cm}$ in longitudinal diameter or greater than $2.5 \mathrm{~cm}$ in vertical diameter, or carrying a cancer history in the same breast with current mass. Written informed consent was obtained before SWE and ultrasound-guided biopsy as the Ethics Committee required. Finally, 119 patients with 122 lesions were recruited in our study.

\subsection{Procedures of SWE and data collection}

All patients were examined with a 4-15 MHz transducer unit of Aixplorer (SuperSonic Imagine, France) by an experienced sonographer, who was just informed with the location of mass and clinical manifestations. Three separate real-time SWE scans swept along the long and the orthogonal short axis, and the images presented in transverse and longitude planes respectively. Masses were in the center of an interest elastic box $\left(3.0 \times 2.5 \mathrm{~cm}^{2}\right.$ in biggest size $)$. All elastic values were demonstrated in $\mathrm{kPa}$ and the default maximum display setting was $180 \mathrm{kPa}$. The quantitative analysis was based on the plane with maximum elastic value across every sweeping, representing the hardest portion of lesions. As showed in Fig. 1., placements of the three quantitative boxes (Qbox, Supersonic Imagine) were suggested: the first $2 \mathrm{~mm}$-diameter round Qbox on the hardest portion (most red on color overlay), the second $2 \mathrm{~mm}$ diameter round Qbox on the softest adjacent parenchyma at the same depth, and the third round Qbox on 
Table 1

Histopathology results of 122 lesions by ultrasound-guided biopsy

\begin{tabular}{lc}
\hline Histopathology & NO. of lesions \\
\hline Malignant $(n=41)$ & 6 \\
Pure ductal carcinoma in situ (DCIS) & 21 \\
Invasive ductal carcinoma (IDC) & 3 \\
Invasive ductal carcinoma + ductal carcinoma in situ & 2 \\
Invasive lobular carcinoma & 1 \\
Invasive ductal carcinoma + invasive lobular carcinoma & 1 \\
Phyllodes tumor & 1 \\
Adenoid cystic carcinoma & 6 \\
Invasive cancer without immunohistochemistry & \\
Benign (n=81) & 37 \\
Fibroadenoma & 23 \\
Adenosis & 4 \\
Fibrocystic changes & 2 \\
Fibroepithelial lesion or phyllodes tumor & 1 \\
Atypical ductal hyperplasia & 3 \\
Papillary lesion & 3 \\
Milk product cyst & 3 \\
Normal tissue & 1 \\
Fibrosis & 1 \\
Cleareen hidradenoma & 1 \\
Foregin body granuloma & 2 \\
Other benign lesion &
\end{tabular}

the whole lesion, including the hardest portion. And quantitative elasticity for the following parameters were analyzed: maximum elasticity (EMAX), mean elasticity of hardest portion (EMANH), mean elasticity of softest adjacent parenchyma (EMEANS), lesion-to-parenchyma elasticity ratio (ERATIO), and mean elasticity and standard deviation of the whole lesion (EMEAN and SD). Finally, histopathology results were obtained.

\subsection{Statistical analysis}

Histopathology results of ultrasound-guided core biopsy were considered as standard of reference. Inter-class correlation coefficient (ICC) was introduced to evaluate reproducibility intra observer. Receiver operation characteristic curve (ROC), area under ROC (Az), sensitivity, specificity, accuracy, and diagnostic index (DI) were introduced to assess diagnostic performance. SPSS 19.0 and MedCalc 14.12.0 were statistical tools we used and a significance level of 0.05 was set.

\section{Results}

\subsection{Histopathology results of all patients}

Histopathology results were obtained after core biopsies under the monitor of B-mode ultrasound, including 41 malignant lesions and 81 benign lesions. The histopathology results are showed in Table 1 .

\subsection{Intraobserver reproducibility of $S W E$}

In longitudinal and transverse planes, EMAX, SD and EMEANH were highly reproducible. The reproducibility of EMAX was high with $\mathrm{ICC}=0.95$ in the transverse plane and ICC $=0.93$ in longitude planes. ERATIO showed lower level of reproducibility. EMEAN and EMEANS were poorly reproduced. 
Table 2

Intraobserver reliability of SWE of 122 lesions

\begin{tabular}{|c|c|c|c|c|c|c|}
\hline \multirow[t]{2}{*}{ Parameter } & \multicolumn{2}{|c|}{ Overall } & \multicolumn{2}{|c|}{ Benign group } & \multicolumn{2}{|c|}{ Malignant group } \\
\hline & ICC & $95 \% \mathrm{CI}$ & ICC & $95 \% \mathrm{CI}$ & ICC & $95 \% \mathrm{CI}$ \\
\hline \multicolumn{7}{|l|}{ SD } \\
\hline Transverse planes & 0.87 & 0.83 to 0.90 & 0.65 & 0.54 to 0.74 & 0.86 & 0.78 to 0.92 \\
\hline Longitude planes & 0.93 & 0.91 to 0.95 & 0.88 & 0.83 to 0.92 & 0.91 & 0.85 to 0.94 \\
\hline \multicolumn{7}{|l|}{ EMAX } \\
\hline Transverse planes & 0.95 & 0.94 to 0.97 & 0.89 & 0.85 to 0.93 & 0.93 & 0.89 to 0.96 \\
\hline Longitude planes & 0.93 & 0.90 to 0.95 & 0.83 & 0.76 to 0.88 & 0.89 & 0.83 to 0.94 \\
\hline \multicolumn{7}{|l|}{ EMEANH } \\
\hline Transverse planes & 0.93 & 0.90 to 0.95 & 0.89 & 0.84 to 0.92 & 0.87 & 0.90 to 0.93 \\
\hline Longitude planes & 0.92 & 0.89 to 0.94 & 0.85 & 0.79 to 0.89 & 0.88 & 0.81 to 0.93 \\
\hline \multicolumn{7}{|l|}{ EMEAN } \\
\hline Transverse planes & 0.49 & 0.38 to 0.59 & 0.24 & 0.10 to 0.38 & 0.80 & 0.70 to 0.88 \\
\hline Longitude planes & 0.88 & 0.84 to 0.91 & 0.84 & 0.77 to 0.89 & 0.87 & 0.79 to 0.92 \\
\hline \multicolumn{7}{|l|}{ EMEANS } \\
\hline Transverse planes & 0.62 & 0.53 to 0.70 & 0.68 & 0.58 to 0.77 & 0.39 & 0.20 to 0.58 \\
\hline Longitude planes & 0.66 & 0.57 to 0.74 & 0.67 & 0.56 to 0.76 & 0.64 & 0.48 to 0.77 \\
\hline \multicolumn{7}{|l|}{ ERATIO } \\
\hline Transverse planes & 0.88 & 0.84 to 0.91 & 0.88 & 0.83 to 0.91 & 0.83 & 0.74 to 0.90 \\
\hline Longitude planes & 0.83 & 0.77 to 0.87 & 0.65 & 0.55 to 0.75 & 0.81 & 0.71 to 0.89 \\
\hline
\end{tabular}

Table 3

Comparison of mass diameter and EMEANS between malignant and benign groups

\begin{tabular}{lccccccc}
\hline \multirow{2}{*}{ Plane } & \multicolumn{3}{c}{$\begin{array}{c}\text { Diameter in B-mode } \\
(\bar{x} \pm s)(\mathrm{mm})\end{array}$} & & & \multicolumn{3}{c}{$\begin{array}{c}\text { EMEANS Median } \\
(\text { Intra-quartile range, IQR) }(\mathrm{kPa})\end{array}$} \\
\cline { 2 - 3 } & Benign & Malignant & $P$ value & & Benign & Malignant & $P$ value \\
\hline Transverse & $11.10 \pm 7.11$ & $13.25 \pm 4.10$ & $>0.05$ & & $6.1(4.4-9.1)$ & $6.6(5.1-8.6)$ & $>0.05$ \\
Longitude & $13.00 \pm 5.48$ & $16.78 \pm 4.60$ & 0.00 & & $6.3(4.8-6.3)$ & $7.5(5.3-9.5)$ & 0.019 \\
Overall & $12.03 \pm 6.39$ & $15.01 \pm 4.68$ & 0.00 & & $6.2(4.6-8.9)$ & $6.9(5.2-9.2)$ & 0.012 \\
\hline
\end{tabular}

Table 4

Comparison of mass diameter and EMEANS within malignant or benign group

\begin{tabular}{|c|c|c|c|c|c|c|}
\hline \multirow[t]{2}{*}{ Group } & \multicolumn{3}{|c|}{ Diameter in B-mode $(\bar{x} \pm s)(\mathrm{mm})$} & \multicolumn{3}{|c|}{ EMEANS Median (IQR) (kPa) } \\
\hline & Transverse & Longitude & $P$ value & Transverse & Longitude & $P$ value \\
\hline Benign & $11.10 \pm 7.11$ & $13.00 \pm 5.48$ & 0.00 & $6.1(4.4-9.1)$ & $6.3(4.8-6.3)$ & $>0.05$ \\
\hline Malignant & $13.25 \pm 4.10$ & $16.78 \pm 4.60$ & 0.00 & $6.6(5.1-8.6)$ & $7.5(5.3-9.5)$ & 0.022 \\
\hline
\end{tabular}

\subsection{Adjacent parenchyma on SWE}

EMEANS presented the stiffness of softest adjacent parenchyma at the same depth with hardest portion in the interest elastic box. Significant differences were observed between benign and malignant group and between orthogonal planes. EMEANS and diameter in B-mode ultrasound showed significantly larger in longitude planes of malignant lesions.

\subsection{Az values}

EMAX, EMEANH and SD had large Az values, and they were significant larger than the values of EMEAN and ERATIO. The mean Az values from different acquisitions with the five parameters were $0.899,0.889,0.887,0.784$ and 0.836 respectively. 
Table 5

Diagnostic performances of EMAX, EMEANH and SD of different planes and different acquisitions

\begin{tabular}{|c|c|c|c|c|c|c|}
\hline Parameter & $\mathrm{Az}$ & Cut-off value $(\mathrm{kPa})$ & Sen $(\%)$ & Spe $(\%)$ & $\operatorname{Acc}(\%)$ & DI $(\%)$ \\
\hline \multicolumn{7}{|l|}{ EMAX } \\
\hline Transverse plane & 0.892 & 50.7 & 78.05 & 92.59 & 86.89 & 170.64 \\
\hline Longitude plane & 0.894 & 45.5 & 78.05 & 90.12 & 85.25 & 168.17 \\
\hline Mean value of orthogonal planes & 0.903 & 43.5 & 82.93 & 88.89 & 86.07 & 171.82 \\
\hline Maximum value of orthogonal planes & 0.897 & 49.1 & 80.49 & 90.12 & 86.07 & 170.61 \\
\hline \multicolumn{7}{|l|}{ EMEANH } \\
\hline Transverse plane & 0.893 & 41.7 & 78.05 & 92.59 & 86.07 & 170.64 \\
\hline Longitude plane & 0.889 & 35.6 & 78.05 & 86.42 & 84.43 & 164.47 \\
\hline Mean value of orthogonal planes & 0.901 & 33.6 & 85.37 & 85.19 & 84.43 & 170.55 \\
\hline Maximum value of orthogonal planes & 0.902 & 43.8 & 80.49 & 90.12 & 86.07 & 170.61 \\
\hline \multicolumn{7}{|l|}{ SD } \\
\hline Transverse plane & 0.875 & 9.5 & 73.17 & 92.59 & 84.43 & 165.76 \\
\hline Longitude plane & 0.891 & 9.9 & 75.61 & 85.06 & 86.07 & 170.67 \\
\hline Mean value of orthogonal planes & 0.888 & 9.0 & 78.05 & 91.36 & 84.43 & 169.41 \\
\hline Maximum value of orthogonal planes & 0.882 & 9.9 & 82.93 & 90.12 & 86.07 & 173.05 \\
\hline
\end{tabular}

\subsection{Diagnostic performances in orthogonal planes}

Diagnostic performance was evaluated after ROC assessment. The associated criterion with highest DI was accepted as cut-off value. Sensitivity, specificity, and accuracy were evaluated with quantitative elasticity from different acquisitions.

Despite EMEAN and ERATIO had diagnostic accuracy lower than 80\%, EMAX, EMEANH and SD showed accuracy more than $84 \%$ (Table 5). The DIs were close to or higher than $170 \%$, meaning the three parameters feasible to apply in diagnosis. Further, the sensitivity had been improved in orthogonal planes without loss of accuracy, though no significant differences were found. Sensitivity of SD rose at from $73.17 \%$ in transverse plane to $82.93 \%$ with maximum SD in orthogonal planes for diagnosis.

\section{Discussion}

In this study, EMAX, EMEANH and SD were high reproducible in real-time acquisition, but ERATIO and EMEAN were not. The ICC values were all larger than previous study [8]. EMAX was most reproducible though no significant differences were found. For real-time continuous scans including elasticity of whole mass and excluding disturbing of slice selection, it is naturally to observe higher reproducibility.

Compared to transverse or longitude plane alone, all parameters had larger Az values in orthogonal planes. But EMEAN and ERATIO showed no clinic feasibility with DI values much less than $170 \%$ (largest with 166.94\%). Based on no loss in accuracy, EMAX, EMEANH and SD were further improved in sensitivity when orthogonal acquisitions conducted. Though the cut-off values are different [1] and some are higher than the values in this study [3]4]11-15], the diagnostic performances are almost fair [3, 4[11-18]. It can be resulted by high rate of DCIS (6/41 in malignant group) and hard rule of avoiding pressure (images with apparent artefacts were excluded) in this study. Several studies have shown that elastic values was different with different immunohistochemical profile and DCISs have lower elasticity value than IDCs [3 4[19]. And the artefact caused by pressure also increases elastic value [4 10].

Even continuous scans in orthogonal planes applied, false negative and false positive cases were found (typical images in Fig. 2). Yoon JH and the colleagues noted that thick breast and large and deep lesions can lead to false positive patterns but early stage cancers can show false negative images [20]. 

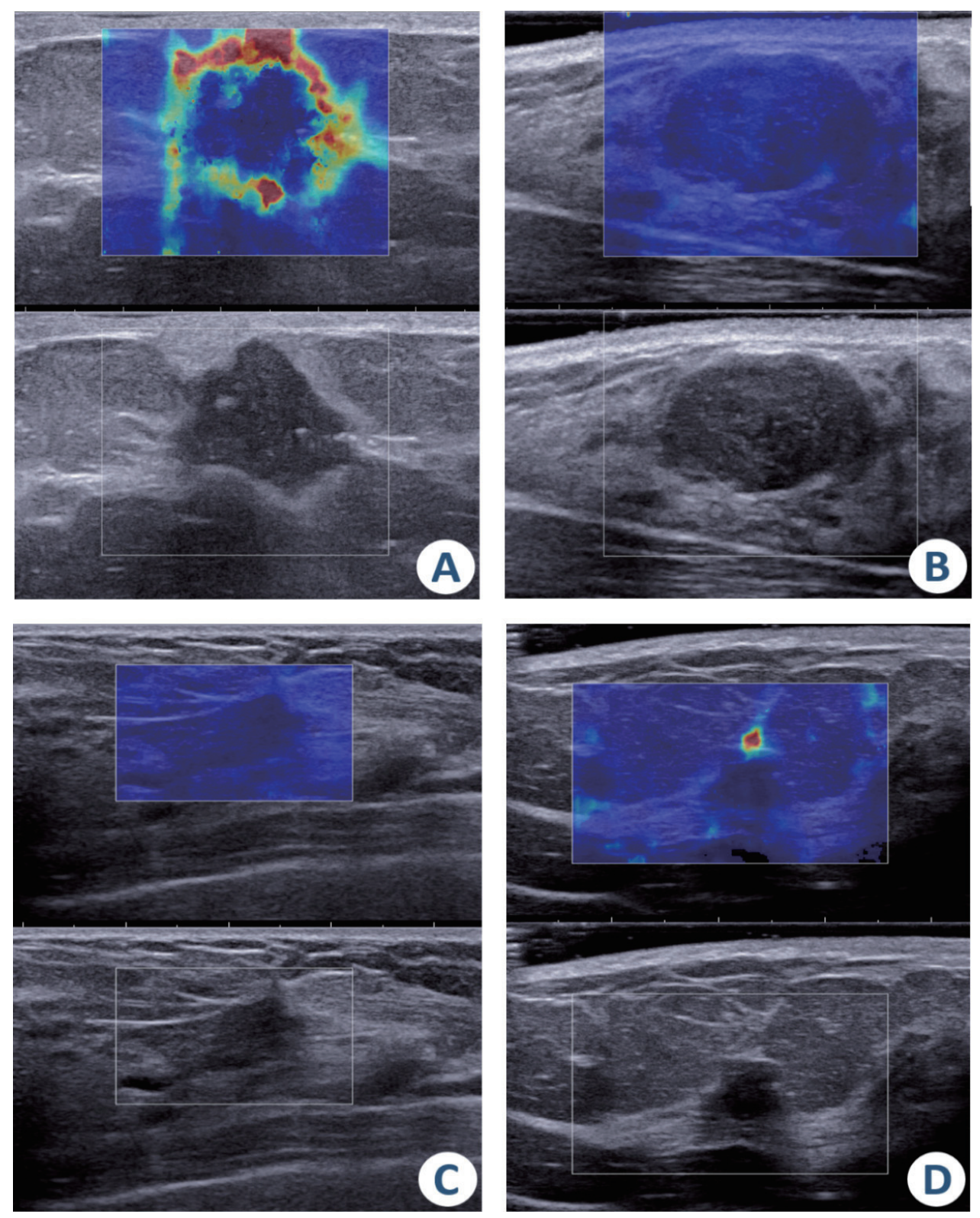

Fig. 2. Figures $2 \mathrm{~A}$ and $\mathrm{B}$ were true positive and true negative cases, and Figs $2 \mathrm{C}$ and $\mathrm{D}$ were false negative and false positive cases. (A) An irregular mass firstly found in a 40-year-old woman. The mass was palpable but not movable. The longitude SWE image showed a stiff halo around the mass with EMAX up to $300.0 \mathrm{kPa}$. Biopsy confirmed invasive ductal carcinoma with ductal carcinoma in situ. (B) A palpable and movable oval mass under annual screening in a 28-year-old woman. The longitude SWE image was homogeneous in light blue with $28.0 \mathrm{kPa}$ in EMAX. It was a fibroadenoma. (C) A palpable and movable mass firstly found in a 45-year-old woman. The mass was irregular in shape and highly suspicious in B-mode ultrasound. The color map of its transverse SWE image was homogeneous with $21.7 \mathrm{kPa}$ in EMAX. But histopathology result showed invasive cancer. (D) A palpable and movable mass firstly found in a 48-year-old woman. The mass was kind of irregular in B-mode and had a stiff dot on transverse SWE image with EMX demonstrating $192.0 \mathrm{kPa}$. It was diagnosed as fibroadenoma after ultrasound-guided biopsy with 16-gauge needle. 
Besides, the diameter and elastic value were found significant larger in longitude plane of malignant lesion. It is known the fact that malignant mass infiltrating surrounding tissue, and the real size can be larger than in B-mode ultrasound. But the interest elastic box was limited in size. It couldn't image healthy breast parenchyma in longitude plane for every recruited malignant lesion, especially the larger or high-grade cancer. So the biggest size of interest elastic box is recommended as default application to decrease the happening of false contrast.

Our study had some limitations. First, the lesion number was small and we did not evaluate the elastography characteristics pf particular histopathology change. Second, the histopathology samples were obtained by core needle biopsies rather than excisions. Third, the combined diagnostic performance of quantitative elasticity was not analyzed. Fourth, the breast imaging reporting and data system (BIRADS) and qualitative elasticity analysis were not included. For further study, the combination of different parameters should be analyzed in a large population.

In conclusion, limited size of elastic image box made infiltrated tissue performing as contrast tissue in longitude plane of some malignant lesions. EMAX, EMEANH, and SD on real-time continuous SWE showed high reproducibility intra observer, and they all had high Az value and accuracy. Compared with examination in single plane, continuous SWE scans in orthogonal planes could improve the sensitivity without loss of accuracy, which is helpful to early diagnosis of breast cancers.

\section{References}

[1] Chen LM, He J, Liu G, et al. Diagnostic performances of shear-wave elastography for identification of malignant breast lesions: a meta-analysis. Jpn J Radiol. 2014; (32):592-599.

[2] Li GL, Li DW, Fang YX, et al. Performance of Shear Wave Elastography for differentiation of Benign and Malignant Solid Breast Masses. PLOS ONE. 2013; 8(10): E76322.

[3] Chang JM, Moon WK, Cho N, et al. Clinical application of shear wave elastography (SWE) in the diagnosis of benign and malignant breast diseases. Breast Cancer Res Treat. 2011; 129:89-97.

[4] Wendie AB, David OC, Caroline JD, et al. Shear-wave elastography improves the specificity of breast US: the BE1 multinational study of 939 masses. Radiology. 2012; 262(2):435-449.

[5] Itoh A, Ueno E, Tohno E, et al. Breast disease: clinical application of US elastography for diagnosis. Radiology. 2006; 239:341-350.

[6] Thomas A, Fischer T, Frey H, et al. Real-time elastography: an advanced method of ultrasound-first results in 108 patients with breast lesions. Ultrasound Obstet Gynecol. 2006; 28:335-340.

[7] Tanter M, Bercoff J, Athanasiou A, et al. Quantitative assessment of breast lesion viscoelasticity: initial clinical results using supersonic shear imaging. Ultrasound Med Biol. 2008; 34:1373-1386.

[8] David O. Cosgrove, Wendie A. Berg, Caroline J Doré, et al. Shear wave elastography for breast masses is highly reproducible. Eur Radiol. 2012; 22:1023-1032.

[9] Athanasiou A, Tardivon A, Tanter M, et al. Breast lesions: quantitative elastography with supersonic shear imagingpreliminary results. Radiology. 2010; 256:297-303.

[10] Youk JH, Gweon HM, Son EJ, et al. Three-dimensional shear-wave elastography for differentiating benign and malignant breast lesions: comparison with two-dimensional shear-wave elastography. Eur Radiol. 2013; 23(6):1519-1527.

[11] Chang JM, Won JK, Lee KB, et al. Comparison of shear-wave and strain ultrasound elastography in the differentiation of benign and malignant breast lesions. Am J Roentgenol. 2013; 201:347-356.

[12] Le EJ, Jung HK, Ko KH, et al. Diagnostic performances of shear wave elastography: which parameter to use in differential diagnosis of solid breast masses? Eur Radiol. 2013; 23(7):1803-1811.

[13] Lee SH, Chang JM, Kim WH, et al. Differentiation of benign from malignant solid breast massed" comparison of twodimensional and three-dimensional shear-wave elastography. Eur Radiol. 2013; 23:1-9.

[14] Yoon JH, Ko KH, Jung HK, et al. Qualitative pattern classification of shear wave elastography for breast masses: how it correlates to quantitative measurements. Rur J Radiol. 2013; 82:2199-2204.

[15] Youk JH, Gweon HM, Son EJ, et al. Three-dimensional shear-wave elastography for differentiating benign and malignant breast lesions: comparison with two-dimensional shear-wave elastography. Eur Radiol. 2013; 23:1519-1527.

[16] Evans A, Whelehan P, Thomoson K, et al. Quantitative shear wave ultrasound elastography: initial experience in solid breast masses. Breast Cancer Research. 2010; 12:R104. 
[17] Evans A, Whelehan P, Thomoson K, et al. Differentiation benign from malignant solid breast masses: value of shear wave elastography according to lesion stiffness combined with greyscale ultrasound sccording to BI-RADS classification. $\mathrm{Br}$ J Cancer. 2012; 107:224-229.

[18] Gweon HM, Youk JH, SON EJ, et al. Visually assessed colour overlay features in shear-wave elastography for breast masses: quantification and diagnostic performance. Eur Radiol. 2013; 23:658-663.

[19] Youk JH, Gweon HM, Son EJ, et al. Shear-wave elastography of invasive breast cancer: correlation between quantitative mean elasticity value and immunohistochemical profile. Breast Cancer Res Treat. 2013; 138(1):119-126.

[20] Yoon JH, Jung HK, Lee JT, et al. Shear-wave elastography in the diagnosis of solid breast masses: what leads to falsenegative or false-positive results? Eur Radiol. 2013; 23(9):2432-2440. 